\title{
Personality and temperamental traits of heterosexual men and MSM
}

\author{
Kurpisz Jacek, Kowalczyk Robert, Nowosielski Krzysztof, Lew-Starowicz Michał, Samochowiec Jerzy
}

According to previous studies (Lippa, 2005), global psychological characteristics differ in groups of various psychosexual orientation. The aim the study was to identify personality and temperament traits differences between heterosexual men and MSM (homo and bisexual) in Poland.

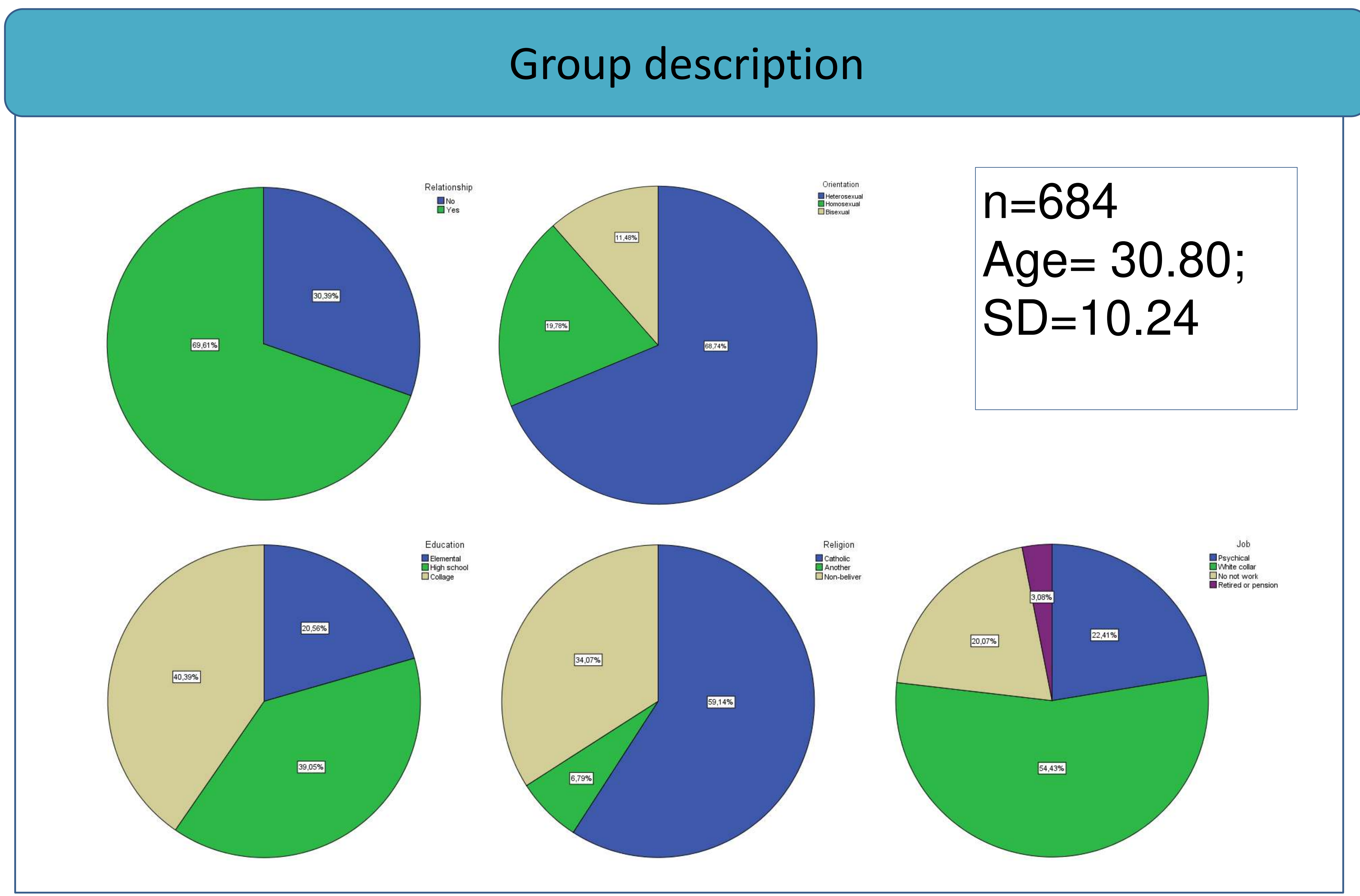
Methods:
$\checkmark$ 'Paper and pencil' and internet survey
$\checkmark$ Examined men filled in questionnaires:
- NEO-FFI (measurement of global personality traits - Big Five - Neuroticism, Extraversion, Openness to experience, Agreeableness, Conscientiousness)
- BIS/BAS (behavioural avoidance/inhibition scales)
- Socio-demographic survey
$\checkmark$ Statistical analysis with Kruskal-Wallis and Mann- Whitney tests with statistical significance level set on $p<0.05$ (Kolmogorov-Smirnov test for all variables: $p$ $<0.05)$

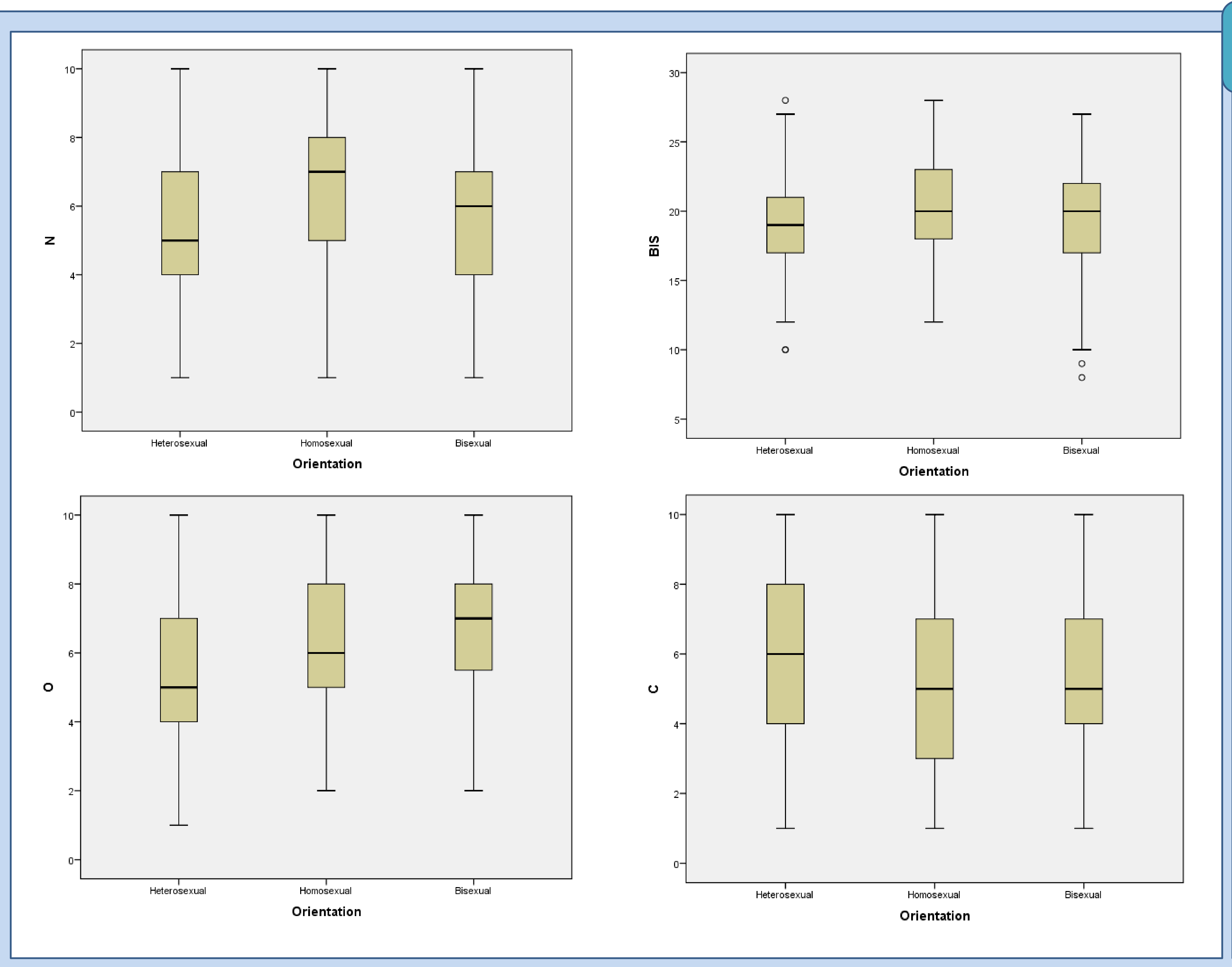

\section{Findings}

Temperamental behavioural activation, measured by BIS/SAS subscales (drive, fun seeking and reward responsiveness) did not differ in examined groups (chi ${ }^{2}$ score for BAS drive was 3.33; for BAS fun seeking 0.46; for BAS reward responsiveness 3.98; all ps>0.05)

$>$ The heterosexual men obtained significantly lower scores than homosexual on behavioural inhibition scale $(z=-3.06, p<0.05)$. No significant differences in BIS between heterosexual and bisexual $(z=-1.13, p>0.05)$ as well as homosexual and bisexual men $(z=-$ $1.24, p>0.05$ ).

$>$ Heterosexual group scored lower on Neuroticism than homosexual $(z=-5.94, p<0.001)$. Bisexual men scored on Neuroticism lower than homosexual $(z=-2.70, p=0.007)$. There was no significant difference between heterosexual and bisexual group on Neuroticism ( $z=-$ $1.78, p>0.05)$.

There were no significant differences between studied groups in relation to Extraversion $\left(\right.$ chi $\left.^{2}=1.42, \mathrm{p}>0.05\right)$ and Agreeableness $\left(\mathrm{chi}^{2}=5.54, \mathrm{p}>0.05\right)$.

- Heterosexual group scored lower on Openness to experience in comparison to homosexual $(z=-5.94, p<0.001)$ and bisexual homosexual $(z=-5.09, p<0.001)$ groups. There was no difference between homosexual and bisexual group $(z=-1.46, p>0.05)$.

$>$ Heterosexual group scored higher on Conscientiousness than homosexual $(z=-2.79$, $p=0.005)$ and bisexual men $(z=-2.14, p=0.03)$. There was no difference between homosexual and bisexual group $(z=-0.19, p>0.05)$.

\section{Conclusions}

Our findings support previous observations, that homosexual men are more neurotic and open to experience in comparison to heterosexual (Lippa, 2005). They are also more prone to behavioural inhibition. This observation could be explained in broad terms of minority stress and availability to live more alternative lifestyle (because of lower pressure of traditional social expectations on gay community). Nevertheless, there were no differences in Extraversion and Agreeableness between those groups, as it was expected according to prior studies (homosexual men usually score lower on Extraversion and higher on Agreeableness). Interestingly, examined heterosexual group was found to be more conscientious than both MSM groups (which is perceived as more stereotypically feminine trait).

Bisexual group was more similar in personality traits description to heterosexual in Neuroticism and behavioural inhibition tendency, while more close to homosexual on Openness to experience and Conscientiousness. Neuroticism and behavioural inhibition scores can reflect, on the one hand, possibility to 'act like heterosexual' and to hide 'gay activities' for relations with women, when heterosexual majority causes any heterosexist pressure. On the other hand, levels of Openness to experience and Conscientiousness can show tendency to feel greater freedom to live more alternative lifestyle and to go beyond traditional male roles. 